\title{
Editorial
}

\section{Endothelium derived relaxing factor/nitric oxide}

Not so long ago the role of the vascular endothelium was unknown. It was considered as a simple monolayer barrier between the blood and the vessel wall. The endothelium is the largest organ in the body and in the last $20 \mathrm{yr}$ its active metabolism has been recognized. It is well known that the endothelium can metabolise catecholamines, 5 hydroxytryptamine and peptides; and is the source of factor 8 , Von Villebrand and plasminogen activator. In the last two decades, the primary role of the endothelium in the control of vascular tone has been established. In 1976, Moncada et al. demonstrated that the endothelial cells produce prostacyclin. ${ }^{1}$ In 1980 Furghgott and Zawadski stressed the obligatory requirement of an intact endothelium in acetylcholine induced relaxation of isolated contracted vascular rings in vitro. ${ }^{2}$ It was the first time that Ach evoked relaxation in vitro. It was then possible to reconcile the effect of Ach in vitro and in vivo where Ach induced a decrease in blood pressure and vascular resistance. Furchgott demonstrated that vascular endothelial cells released a vasodilator which was called EDRF (endothelium derived relaxing factor) since the nature of this substance was unknown. Rapidly it became evident that EDRF could be released not only by Ach but my many other substances and physico-chemical stimuli applied to endothelial cells. In 1986 Furchgott suggested that EDRF could be nitric oxide, an unstable gas with a very short half-life. Several authors have confirmed that EDRF is NO or a substance containing NO. ${ }^{3}$ At the end of the eighties it became evident that the endothelium released other vasoactive substances, either vasodilators like EDHF (endothelium derived hyperpolarising factor) ${ }^{4}$ or vasoconstrictors (prostanoids, superoxide anion, endothelin). ${ }^{5}$

Why is a review article necessary in an anaesthesia journal on EDRF/NO, which is only one of the multiple vasoactive substances produced by the endothelium? Why is it important for the anesthetist to be aware of the role of EDRF/NO? Why is the review by Searle and Sahab so timely?

University of Montreal, Notre-Dame Hospital, C.P. 1560, Succursale C, Montreal, Quebec, Canada H2L 4K8.
It is essential that anaesthetists differentiate NO, nitric oxide, from $\mathrm{N}_{2} \mathrm{O}$, nitrous oxide. Nitrous oxide is a very stable compound which is not metabolised and does not react with $\mathrm{O}_{2}$ or haemoglobin. On the other hand, NO is not an anaesthetic, it is a gas which is produced as a very short-acting free radical with a high affinity for $\mathrm{O}_{2}$ and for the haeme moity of haemoglobin and other haeme containing proteins like soluble guanylate cyclase.

The major role of NO in cardiovascular physiology is well established: the control of systemic and pulmonary vascular tone, myocardial contraction, flow distribution, platelet aggregation, smooth muscle cell growth, ... . More recently, NO has been recognized as an important intermediate in inter-cell communication and cell control. ${ }^{6} \mathrm{Nitric}$ oxide has a role in immunology (control of macrophage function and graft rejection ${ }^{7}$ ); it is a central and a peripheral neurotransmittor. ${ }^{8}$ Some anaesthetic agents can interfere with endothelial cell function; local anaesthetics ${ }^{9}$ and volatile anaesthetics ${ }^{10}$ can inhibit the endothelium dependant relaxation. The importance of this inhibition on the general physiology is not known.

The nitrovasodilators, like nitroglycerin and Na nitroprusside release NO intracellularly, which stimulates soluble guanylate cyclase, increases cyclic GMP and induces relaxation. New medications, also in vivo NO liberators, are already on the European market or in the development process.

Nitric oxide itself, in gaseous form, has already been used therapeutically: inhaled NO at a low concentration (40 ppm) is a selective pulmonary artery vasodilator with the same potency as other pulmonary vasodilators such as nitroglycerin and prostacyclin but without any systemic effect. ${ }^{11}$ Low concentrations of $\mathrm{NO}$ are necessary to avoid excessive oxidation of $\mathrm{NO}$ to nitrite, $\mathrm{NO}_{2}$, which is toxic to the lung: close monitoring of $\mathrm{FI} \mathrm{NO}$ and $\mathrm{FI} \mathrm{NO}$, has to be provided. Nitric oxide could be very useful in pulmonary hypertension following cardiac valvular surgery ${ }^{12}$ and cardiac transplantation, in pulmonary hypertension following reversal of heparin with protamine. Zapol and his team in Boston have used inhaled NO with success in newborns with persistant pulmonary hypertension and with 
congenital diaphragmatic hernia. They insisted that this therapy avoided the one of an extra corporeal membrane oxygenator (Zapol W., personal communication at the meeting "Nitric Oxide, Implications for drug research," June 1992, Philadelphia, PA). In ARDS there is a pulmonary hypertension and a mismatching of the ventilation and perfusion. Inhaled NO could reduce the pulmonary vascular pressure and increase the ventilation: perfusion ratio as, because of its localised action, it will dilate only the pulmonary vessels perfusing the ventilated alveolae. Falke, in Berlin, already has considerable experience with this new therapeutic technique in ARDS.

Septic shock is a condition in which a large amount of NO is released not only from the endothelial cells but also from the mastocytes, the smooth muscle cells and the kidney; this is due to the induction of a very productive non-calcium dependant NO synthase. ${ }^{13}$ The excess NO is responsible for hypotension ${ }^{14}$ and myocardial depression. ${ }^{15}$ Inhibitors of NO synthase may have a therapeutic role in this pathology. If some blockade of NO synthase is beneficial, complete blockade is dangerous as it can rapidly kill the animal in septic shock. ${ }^{16}$

Nitric oxide will be used as a therapeutic agent very soon in the OR and intensive care; NO synthase blockers will probably be used in intensive care a little later; for both of these new therapeutic approaches, the anaesthetist will be in the front line, which makes the review by Serale and Sahab so timely.

As NO synthase has been found in the cerebellum, ${ }^{17}$ central $^{18}$ and peripheral ${ }^{19}$ nervous system, NO is recognized as a new class of neurotransmittor. The neurons can release a gas which can diffuse and interact with several others structures. Stimulation of NMDA receptors by glutamate releases NO and increases cyclic GMP in target cells. ${ }^{20}$ The role of NO in normal neurophysiology and in some neurological pathology such as epilepsy, stroke, Alzeimer disease, pain transmission, long-term depression, long-term potentialisation, and memory is more established. $^{21-23}$

In the near future the role of several neurotropic and neurodepressant agents on neurological NO synthase (NOS) pathway will be known. This field of research will be of major interest for the anaesthetist. Indeed R. Johns (personal communication at the meeting "Nitric Oxide, Implications for drug research," June 1992, Philadelphia, PA) has recently shown that the blockade of NOS by LNMMA reduces MAC, and Meller et al. ${ }^{24}$ have shown that stimulation of NMDA receptors at the spinal level produces facilatation of the nociceptive tail flick reflex, which is mediated through the NO cyclic GMP pathways.

As NO is a gas with a very short half-life, the techniques of in situ direct NO measurement are difficult. This tool is, however, very useful for the testing of the new medications that are being developed to modulate the EDRF/NO pathways. This field of research is one of the fastest-growing fields in physiology. It has and will affect anaesthesia even more in the near future.

\section{Le facteur relaxant endothélial et le monoxyde d'azote}

Il n'y a pas si longtemps, on connaissait très peu les fonctions de l'endothélium; on le considérait comme une couche de cellules, située entre le sang et la paroi musculaire du vaisseau. Pourtant l'endothélium constitue l'organe le plus volumineux de notre anatomie et ce n'est qu'au cours des vingt dernières années qu'on lui a découvert un métabolisme actif. Il est bien connu maintenant que l'endothélium peut métaboliser les catécholamines, la 5-hydroxytryptamine et certains peptides. Il synthétise le facteur VIII, le facteur de Von Willebrand ainsi que l'activateur du plasminogène. Pendant ces deux décennies, on a aussi découvert à l'endothélium un rôle primordial dans le contrôle du tonus vasculaire. En 1976, Moncada et coll. ont montré que les cellules endothéliales sécrétaient de la prostacycline. ${ }^{1}$ En 1980 , Furchgott et Zawadski démontraient qu'il fallait absolument la présence d'un endothélium intact pour que l'acétylcholine (Ach) relaxe des anneaux vasculaires préalablement contractés in vitro. C'était la première fois qu'on obtenait une relaxation vasculaire de cette façon. ${ }^{2}$ Il devenait alors possible de réconcilier les effets in vitro de l'Ach avec ses effets in vivo où l'Ach diminue la pression artérielle et entraîne une vasodilatation périphérique. Comme Furchgott ignorait la composition chimique de la substance vasodilatatrice produite par les cellules endothéliales, il l'appela EDRF (endothélium derived relaxing factor). On se rendit rapidement compte que l'EDRF était non seulement sécrété après une stimulation cholinergique mais, que de nombreux autres agonistes et stimuli physicochimiques agissant sur les cellules endothéliales, libéraient aussi de l'EDRF. En 1986, Furchgott suggérait que l'EDRF était du monoxyde d'azote (NO), un gaz très instable à demi-vie très courte. De nombreux auteurs ont confirmé par la suite que l'ERDF est le monoxyde d'azote ou une substance contenant du NO. ${ }^{3} \AA \grave{A}$ la fin des années quatre-vingt, on a découvert que l'endothélium sécrétait aussi d'autres 
substances vasodilatatrices telles que l'«endothelium derived hyperpolarising factor ${ }^{4}$ ou vasoconstrictrices telles que les prostanoïdes, l'anion superoxyde et l'endothéline. 5

Pourquoi une revue sur l'EDRF/NO dans un journal d'anesthésie alors que le monoxyde d'azote ne représente qu'un des nombreux vasodilatateurs sécrétés par l'endothélium ? Pourquoi les anesthésistes doivent-ils se familiariser avec le rôle de l'EDRF/NO et pourquoi le tour d'horizon que nous proposent Searle et Sahab arrive-t-il à point ?

Il est d'abord essentiel de ne pas confondre le monoxyde d'azote avec le protoxyde d'azote $\left(\mathrm{N}_{2} \mathrm{O}\right)$, le gaz analgésique que nous administrons quotidiennement. Le $\mathrm{N}_{2} \mathrm{O}$ est très stable, n'est pas métabolisé dans l'organisme et ne réagit pas avec l'oxygène, ni avec l'hémoglobine. Le monoxyde d'azote n'a pas de propriétés anesthésiques ; il s'agit d'un gaz composé d'un radical libre ayant une demi-vie très courte et une très grande affinité pour l'oxygène, pour l'hème de l'hémoglobine et d'autres protéines comme la guanylate cyclase soluble.

Le rôle fondamental du monoxyde d'azote en physiologie cardiovasculaire est connu. Parmi ses fonctions principales, il faut noter le contrôle du tonus vasculaire de la circulation pulmonaire et systémique, le contrôle de la contractilité myocardique, de la distribution des débits sanguins locaux, de l'inhibition de l'aggrégation plaquettaire et de la croissance des fibres musculaires lisses, etc. Plus récemment, on a découvert que le monoxyde d'azote agissait comme un mediateur important du contrôle cellulaire et comme agent de communication intercellulaire. ${ }^{6}$ En immunologie, le monoxyde d'azote contrôle la fonction bactéricide des macrophages et joue un rôle dans le phénomène de rejet des greffes. ' En neurologie, il est considéré comme un neurotransmetteur du système nerveux central et périphérique. ${ }^{8}$ Certains anesthésiques influencent l'activité des cellules endothéliales. Les anesthésiques locaux ${ }^{9}$ et volatils ${ }^{10}$ inhibent la vasodilatation dépendante de l'endothélium mais le rôle physiologique de cette inhibition n'a pas été élucidé jusqu'à ce jour.

Les dérivés nitrés tels la nitroglycérine et le nitroprussiate de soude, agissent en libérant du monoxyde d'azote intracellulaire ; celui-ci active la guanylate cyclase soluble, augmente le GMP cyclique et induit la relaxation. Plusieurs nouveaux médicaments qui libèrent aussi du monoxyde d'azote sont en voie développement ou ont déjà atteint le marché européen. Le monoxyde d'azote sous sa forme gazeuse a déjà été utilisé en thérapeutique humaine. A faible concentration (40 PPM), le gaz devient un vasodilatateur sélectif de la circulation pulmonaire, et possède la même puissance que les autres vasodilatateurs pulmonaires en usage comme la nitro- glycérine et la prostacycline. Il n'a pas d'effet systémique. " Il faut utiliser une faible concentration de monoxyde d'azote pour éviter l'oxydation trop importante du $\mathrm{NO}$ en nitrite $\left(\mathrm{NO}_{2}\right)$ lequel est toxique pour les pneumocytes. Le monitorage de la fraction inspirée de monoxyde d'azote (FINO) et de $\mathrm{NO}_{2}\left(\mathrm{FINO}_{2}\right)$ est indispensable. Le monoxyde d'azote pourrait être très utile pour traiter l'hypertension pulmonaire après une chirurgie valvulaire cardiaque ${ }^{12}$ et après une transplantation cardiaque et pour traiter l'hypertension pulmonaire consécutive à la neutralisation de l'héparine par la protamine. W. Zapol et son équipe de Boston ont traité au protoxyde d'azote des nouveaux-nés porteur du syndrome de persistance de la circulation foetale et de hernie diaphragmatique. Ils sont convaincus que cette modalité thérapeutique a permis d'éviter un traitement par oxygénateur à membrane (Zapol W., communication au congrès sur « Nitric oxide, Implications for drug research » Juin 1992, Philadelphie).

Le syndrome de détresse respiratoire de l'adulte (ARDS) est caractérisé par une hypertension pulmonaire et une perturbation du rapport ventilation-perfusion. Le monoxyde d'azote en inhalation pourrait réduire l'hypertension pulmonaire et améliorer le rapport ventilation/perfusion dans l'ARDS par son action localisée qui lui permet de dilater uniquement les vaisseaux perfusant les alvéoles ventilées. Le Dr Conrad Falke de Berlin a déjà une expérience clinique avec cette thérapeutique dans l'ARDS.

Dans le choc septique, il y a sécrétion massive de monoxyde d'azote non seulement par les cellules endothéliales mais par les mastocytes, les fibres musculaires lisses et les cellules rénales. Cette sécrétion de monoxyde est due à l'induction d'une NO synthétase très efficace et indépendante du $\mathrm{Ca}^{++} \cdot{ }^{13}$ L'excès de monoxyde d'azote synthétisé est responsable de l'hypotension ${ }^{14}$ et de la dépression myocardique. ${ }^{15}$ Les inhibiteurs de la NO synthétase pourraient trouver une place dans la thérapie du choc septique ; si un blocage partiel de la NO synthétase est bénéfique, un blocage complet est dangereux et peut faire mourir rapidement de choc septique l'animal de laboratoire. ${ }^{16}$ L'utilisation des bloqueurs de la NO synthétase dans traitement du choc septique demeure donc toujours au stade de l'expérimentation.

Le monoxyde d'azote sera utilisé comme agent thérapeutique sous peu en salle d'opération et à l'unité de soins intensifs ; suivront plus tard aux soins intensifs, les bloqueurs de la NO synthétase. Comme chacune de ces nouvelles approches thérapeutiques concerne directement les anesthésistes, le tour d'horizon proposé par Searle et Sahab ne pourrait nous parvenir à un meilleur moment.

Comme on a aussi découvert du monoxyde d'azote au 
niveau du cervelet, ${ }^{17}$ du système nerveux central ${ }^{18}$ et périphérique, ${ }^{19}$ on considère que le monoxyde d'azote appartient à nouvelle classe de neurotransmetteurs. Les neurones sécrètent un gaz qui diffuse et entre en contact avec les autres structures. La stimulation du récepteur NMDA par le glutamate libère du monoxyde et augmente le taux de GMP cyclique dans les cellules cibles. ${ }^{20}$ On attribue de plus en plus un rôle au monoxyde d'azote en physiologie nerveuse comme dans la transmission de la douleur, la potentialisation et la dépression à long terme de la transmission nerveuse ainsi que dans la mémoire et dans certaines pathologies nerveuse comme l'épilepsie, l'accident cérébrovasculaire, la maladie d'Alzheimer. ${ }^{21-23}$

Dans un avenir prochain, nous découvrirons l'influence de nombreuses substances neurotropes et sédatives sur la voie transmission de la NO synthétase. Ce champ de recherche sera d'un grand intérêt pour les anesthésistes. Déjà R. Johns (communication personnelle à la réunion de juin 1992 à Philadelphie) a montré que le blocage de la NO synthétase diminue le MAC chez l'animal et Meller et coll. ${ }^{24}$ ont montré qu'au niveau médullaire, la stimulation du récepteur NMDA produit une facilitation du réflexe nociceptif du mouvement de la queue de rat (tail flick reflex). Cette facilitation est transmise par la voie monoxyde d'azote - GMP cyclique. Comme le monoxyde d'azote existe à l'état de gaz à demi-vie très courte, les techniques de mesure directe du NO in vivo se heurtent à des difficultés importantes. L'élaboration d'une technique de mesure in vivo à la fois sensible et simple s'impose pour rendre possible l'étude des effets de nouvelles drogues qui bloquent la voie métabolique du monoxyde d'azote. Ce champ de recherche physiologique a pris son envoi et évolue très rapidement. $L$ 'anesthésiologie profite déjà des ces découvertes et tout porte à croire que ceci ne fait que débuter.

\section{References}

1 Moncada S, Gryglewski R, Bunting S, Vane JR. An enzyme isolated from arteries transforms prostaglandin endoperoxides to an unstable substance that inhibits platelet aggregation. Nature 1976; 663-5.

2 Furchgott RF, Zawadzki $J$. The obligatory role of endothelial cells in the relaxation of arterial smooth muscle by acetylcholine. Nature 1980; 288: 373-6.

3 Myers PR, Minor RL, Guerra R Jr, Bates JN, Harrison $D G$. Vasorelaxant properties of the endothelium-derived relaxing factor more closely resemble S-nitrosocysteine than nitric oxide. Nature $1990 ; 345: 161-3$.

4 Feletou M, Vanhoutte $P M$. Endothelium-dependent hyperpolarization of canine coronary artery smooth muscle. Br J Pharmacol 1988; 93: 515-24.
5 Luscher TF, Boulanger CM, Dohi Y, Yang Z. Endothelium-derived contracting factors. Hypertension 1992; 19 : 117-30.

6 Moncada S, Palmer RMJ, Higgs EA. Biosynthesis of nitric oxide from $\mathrm{L}$-arginine. A pathway for the regulation of cell function and communication. Biochem Pharmacol 1989; 38: 1709-15.

7 Marletta MA. Nitric oxide: biosynthesis and biological significance. Trends in Biological Sciences 1989; 14: 488-92.

8 Bredt DS, Snyder SH. Nitric oxide, a novel neuronal messenger. Neuron 1992; 8: 3-11.

9 Johns RA. Local anesthetics inhibit endothelium-dependent vasodilation. Anesthesiology 1989; 70: 805-11.

10 Uggeri MJ, Proctor GJ, Johns RA. Halothane, enflurane, and isoflurane attenuate both receptor- and non-receptormediated EDRF production in rat thoracic aorta. Anesthesiology 1992; 76: 1012-7.

11 Frostell C, Fratacci MD, Wain JC, Jones $R$, Zapol WM. Inhaled nitric oxide. A selective pulmonary vasodilator reversing hypoxic pulmonary vasoconstriction. Circulation 1991; 83: 2038-47.

12 Girard C, Lehot JJ, Clerc J, Ffrech P, Estanove S. Inhaled nitric oxide (NO) in pulmonary hypertension following mitral valve replacement. Anesthesiology 1991; 75, A984.

13 Forstermann U, Schmidt HHHW, Pollock JS, et al. Isoforms of nitric oxide synthase. Characterization and purification from different cell types. Biochem Pharmacol 1991; 42: 1849-57.

14 Kilbourn RG, Jubran A, Gross SS, et al. Reversal of endotoxin-mediated shock by $N^{G}$-methyl-L-arginine, an inhibitor of nitric oxide synthesis. Biochem Biophys Res Commun 1990; 172: 1132-8.

15 Schulz R, Nava E, Moncada S. Induction and potential biological relevance of a $\mathrm{Ca}^{2+}$-independent nitric oxide synthase in the myocardium. Br J Pharmacol 1992; 105 : 575-80.

16 Wright, et al. LNNMA administration before or after LPS-induced shock enhances hypotension, organ hypoperfusion and mortality in anesthetized rabbits. Cardiovasc Res 1992; 26: 48.

17 Bredt DS, Snyder SH. Nitric oxide mediates glutamatelinked enhancement of cGMP levels in the cerebellum. Proc National Academy of Science USA 1989; 86 : 9030-3.

18 Garthwaite J, Garthwaite G, Palmer RMJ, Moncada S. NMDA receptor activation induces nitric oxide synthesis from arginine in rat brain slices. Eur J Pharmacol 1989; 172: 413-6.

19 Bult H, Boeckxstaens GE, Pelckmans PA, Jordaens FH, Van Maercke YM, Herman AG. Nitric oxide as an inhibitory non-adrenergic non-cholinergic neurotransmitter. Nature $1990 ; 345$ : 346-7. 
20 Hoffman $M$. A new role for gases: neurotransmission. Science 1991; 252: 1788.

21 Dawson TM, Dawson VL, Snyder SH. Neurological progress; a novel neuronal messenger molecule in brain: the free radical, nitric oxide (NO). Ann Neurol 1992 (in press).

22 Snyder SH, Bredt DS. Biological roles of nitric oxide. This previously elusive and obscure chemical is proving to be of vital physiological significance. Nitric oxide may be the first of a novel class of neurotransmitters. Scientific American May 1992; 68-77.

23 McCall T, Vallance $P$. Nitric oxide takes centre-stage with newly defined roles. TIPS 1992; 13: 1-5.

24 Meller ST, Dykstra C, Gebhart GF. Production of endogenous nitric oxide and activation of soluble guanylate cyclase are required for $\mathrm{N}$-methyl-D-aspartate-produced facilitation of the nociceptive tail-flick reflex. Eur J Pharmacol 1992; 214: 93-6. 